\title{
The Experimental Study of the Influence of Yanlingdan Prescription on the Apoptosis Protein Fas/FasL Expression of Ovarian Granular Cells in Mice with Premature Ovarian Failure
}

\author{
Yang Wang ${ }^{1 \mathrm{a}}$, Penghui $\mathrm{Li}^{2 \mathrm{~b}}{ }^{*}$, Cuiping $\mathrm{Li}^{3 \mathrm{c}}$, Bin Wang ${ }^{4 \mathrm{~d}}$ Shudong Niu${ }^{5}$ e , Libo \\ Wen $^{6 \mathrm{f}}$, Kun Zhao ${ }^{7} \mathrm{~g}$, Yuge Wang ${ }^{8 \mathrm{~h}}$ and Yuefei Wang ${ }^{9 \mathrm{i}}$ \\ 1,2Department of Physiology of Qiqihar Medical University, Qiqihar, Heilongjiang, China,161006 \\ ${ }^{3}$ The Third Affiliated Hospital of Qiqihar Medical University, gynecology and obstetrics, \\ China,161006 \\ 4,5,6,7,8,9 Department of Physiology of Qiqihar Medical University, Qiqihar, Heilongjiang, \\ China,161006 \\ aRachal830813@qq.com, b936349522@qq.com, c153266751@qq.com, dicao7835@sina.com,

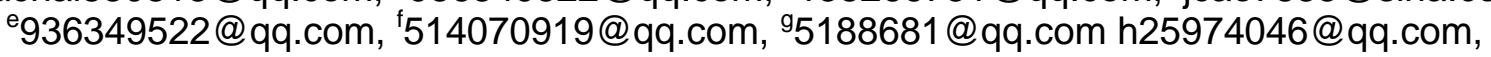 \\ ijbmwyg08@163.com
}

Keywords: Yanlingdan prescription; Granular cells; Apoptosis proteins; FAS; FASL

\begin{abstract}
Objective: The purpose of this research is to observe the influence of Yanlingdan prescription on the apoptosis protein Fas/FasL expression of ovarian granular cells in mice with autoimmune premature ovarian failure and investigate the mechanism of this prescription in the treatment of premature ovarian failure. Method: Taking the Zona pellucida3 of mice as the antigen, the female mice were injected subcutaneously with this drug to make mice immune so as to become the mice with premature ovarian failure. Blank control group, model group, Yanlingdan group and western medicine group are set. After two weeks of dosing mice, we use immunohistochemical method to detect the expression level of apoptosis related protein Fas/FasL of ovarian granular cells. Results: The expression level of apoptosis related protein Fas/FasL in model group is obviously higher than the blank control group $(\mathrm{P}<0.01)$. Compared with model group, the FAS and FASL protein expression of mice' follicle in Yanlingdan group and western medicine group weakens obviously $(\mathrm{P}<0.01)$. When the expression of Fas and Fasl in Yanlingdan group compares with the normal control group, the difference has no statistical significance $(\mathrm{P}>0.05)$. Conclusion: Yanlingdan prescription can effectively suppress the expression of FAS/FASL protein of follicular granular cells and have inhibitory effects on the apoptosis of ovarian granular cells in mice with premature ovarian failure.
\end{abstract}

\section{Introduction}

Premature Ovarian Failure (POF) refers to the ovarian function failure which is resulted by follicular atresia before the age of 40. The clinical manifestations of patients are the high gonadotropin level, low oestrogen level, amenorrhoea, infertility and various perimenopausal period symptoms. With the change of the living environment, dietary habit and work stress of modern society, the morbidity of premature ovarian failure has increased gradually[1]. Modern research shows that[2] the excessive apoptosis of ovarian granular cells is one of the main reasons to lead to premature ovarian failure. Ovarian granular cells can not only secrete estrogen and progestational hormone but also play an important role in the process of development and maturity of follicle, therefore the adjustment of the apoptosis of follicular granular cells may provide new target spot for the treatment of premature ovarian failure. Through the animal experiment, this research explores the influence of Yanlingdan prescription on the molecular regulation mechanism of the apoptosis pathway which is mediated by Fas in ovarian granular cells and investigates the mechanism of action of this prescription. 


\section{Materials and Methods}

Animal. The animal center of Qiqihar Medical University provides 7-8 weeks old 60 SPF BABL/C female mice and 10 male mice. The weight of mice is $18 \sim 22 \mathrm{~g}$. The certification number of the animal is $\operatorname{SCXK}$ (black)2015-0004. Females and males were reared in separate rearing cages at a rate of 4 to 1 in order to induce normal estrous cycles in mice. Exfoliated cells of the vagina were made into smears every day in order to observe the estrus cycle of mice under the microscope. Mice with regular estrous cycle are selected to conduct the experiment (The calculation is based on two estrous cycles).

Experimental Medicine. The first one is traditional Chinese medicine preparations.Yanlingdan prescription: rehmannia $12 \mathrm{~g}$, Chinese yam $12 \mathrm{~g}$, sweet potato $12 \mathrm{~g}$, Chinese dodder $12 \mathrm{~g}$, Eucommia ulmoides Oliver $12 \mathrm{~g}$, fleece-flower root $6 \mathrm{~g}$, black ants $6 \mathrm{~g}$, oyster $6 \mathrm{~g}$, the fruit of Chinese wolfberry $6 \mathrm{~g}$, the root of red-rooted salvia $6 \mathrm{~g}, 6$ Cyperus rotundus, root of membranous milk vetch $6 \mathrm{~g}$, Codonopsis pilosula $6 \mathrm{~g}$, liquorice $3 \mathrm{~g}$. These medicines are provided by the bureau of traditional Chinese medicine in Qiqihar. The dosage form is granules. And it is made into the solvent which is equivalent to $1.08 \mathrm{~g} / \mathrm{L}$ crude drugs. It should be reserved in the refrigerator of $4^{\circ} \mathrm{C}$. The second one is western medicine. Estradiol Valerate Tablets which are provided by Guangzhou Branch of Bayer Medicine and Health Care Co.,Ltd. Each tablet is $1 \mathrm{mg}$. It is diluted by tri-distilled water. The concentration of it is $0.006 \mathrm{mg} / \mathrm{ml}$.

Kit. The zona pellucida polypeptide solution of mice: The 330th 342th amino acid sequence (NSSSSQFQIHGPR) of the zona pellucida3(ZP3) of mice. The purity of it is greater than 95\%. It is produced by Chinese Peptide Co.,Ltd. Freund's complete adjuvant and freund's incomplete adjuvant which are provided by Beijing Bioss Biotechnology Co.,Ltd. FAS and FASL primary antibodies which are provided by Wuhan Boster Biological Technology Co.,Ltd.

Experimental Instruments. BX53 optical microscope (Japanese Olympus Company). RM2235 freezing microtome (German Lecai company).

\section{Methods}

POF Model Equipment. Tri-distilled water is added to $2 \mathrm{mg} \mathrm{ZP} 3$ zona pellucida polypeptide powder to make into the solution. The selected $1 \mathrm{ml}$ solution is diluted until $6 \mathrm{ml}$ solution. Then solution and freund's complete adjuvant are made into the immunoreagent at a ratio of 1:1. Solution and freund's incomplete adjuvant are made into the immunopotentiator at a ratio of 1:1. Model is made according to the literature method[3]. Double rear soles and abdominal cavity of mice are subcutaneously injected $0.15 \mathrm{ml}$ immunoreagent. After 14 days, double rear soles and abdominal cavity of mice are subcutaneously injected $0.15 \mathrm{ml}$ immunopotentiator to strengthen immunity.

Groups and Feeding Drug. 40 mice with regular estrous cycle are divided into blank control group, model group, Yanlingdan group and western medicine group. In addition to the blank control group, double rear soles and abdominal cavity of mice in model and other two groups are subcutaneously injected $0.15 \mathrm{ml}$ immunoreagent. The mice in the blank control group are injected $0.15 \mathrm{ml}$ normal saline. After 14 days, double rear soles and abdominal cavity of mice in model and other two groups are subcutaneously injected $0.15 \mathrm{ml}$ fresh immunopotentiator. The mice in the blank control group are interjected $0.15 \mathrm{ml}$ normal saline again. Yanlingdan prescription group was given 2 times clinical equivalent dosage $(1.08 \mathrm{ml} / 20 \mathrm{~g})$ Yanlingdan prescription liquor by gavage. The western group is given $0.4 \mathrm{ml} / 20$ gestradiol valerate by gavage. The blank group and model group are given normal saline by gavage. Each group is given twice a day for 15 days.

Observation Index. The first one is estrous cycle observation. The estrous cycle of autoimmune POF model mice is observed[4]. Vaginal cells smears are inspected at 9:00 every morning in order to observe and compare the changes of the estrous cycle of mice in each group. The judgement basis of the estrous cycle: There are a large number of nucleated epithelial cells and a small amount of keratinized epithelial cells in the prophase of estrus. There are a large number of keratinized epithelial cells in estrus. In the late stage of estrus, there are mainly keratinized epithelial cells accompanied by a few white blood cells. There are a large number of leukocytes during diestrus. 
The second one is ovarian Fas and Fasl proteins expression detection. After the mice were killed, aseptic ovarian tissues were reserved. They are fixed by paraformaldehyde and embedded and cut into slices by paraffin. The expression of Fas and FasL proteins in the ovary was detected by immunohistochemistry according to the kit instruction. The third one is a statistical method. SPSS18.0 statistical software is used. Measurement data is expressed by using mean \pm standard deviation $(x \pm s)$. Analysis of variance was used in multiple groups. SNK method is used in the comparison between two groups. The difference has statistical significance $(\mathrm{P}<0.05)$.

\section{Results}

Comparison in General Condition. The mice in the blank group have glossy fur, good eating, regular bowel movements, quick movement and quick reaction. The fur of mice in model group fur is lackluster and sparse, erect. They eat very little. Their stool is thin. They are tired, apathetic. They have less independent activities and like to stay with each other and curl up.

Comparison in Estrous Cycle. The estrous cycle of normal mice is $4 \sim 5$ days. The diestrus is 1.5 2.5 days. After the model is made, the estrous cycle of mice in the model group is prolonged. The period appears irregular disorder and the cycle was prolonged to 7 10 days. Most of them stay at diestrus. The disorder order of the estrous cycle of mice in Yanlingdan treatment group is improved. There is a regular estrous cycle alternation and the time is $6 \sim 9$ days.

The Influence of Yanlingdan Prescription on the Expression of Fas and FasL Proteins of Follicular Granular Cells in Mice with Autoimmune Premature Ovarian Failure. The expression of FAS protein of follicular granular cells of mice in blank control group is little and the expression of FASL protein is weakly positive. Compared with the blank group, the expression of FAS and FASL proteins of follicular granular cells of mice in the model group is obviously increased $(\mathrm{P}<0.01$ ) . Compared with the model group, the expression of FAS and FASL proteins of follicular granular cells of mice in Yanlingdan group and western medicine group is obviously decreased $(\mathrm{P}<0.05)$. It shows that Yanlingdan group and western medicine group both can restrain the excessive apoptosis of follicular granular cells through decreasing the expression of FAS and FASL proteins of follicular granular cells of mice.

Table 1. The influence of the Wubie oral liquid on the expression of ovarian FAS/FASL protein of mice with autoimmune premature ovarian failure.

\begin{tabular}{|c|c|c|c|c|}
\hline Groups & $\begin{array}{c}\text { The number of } \\
\text { ovaries }\end{array}$ & FAS & FASL & \\
\hline $\begin{array}{l}\text { Blank control } \\
\text { group }\end{array}$ & 10 & $0.200 \pm 0.008$ & $1.532 \pm 0.441$ & \\
\hline Model group & 10 & $0.468 \pm 0.021 \quad \Delta$ & $2.344 \pm 0.345$ & $\boldsymbol{\Delta}$ \\
\hline $\begin{array}{l}\text { Yanlingdan } \\
\text { group }\end{array}$ & 10 & $0.208 \pm 0.141 \square \nabla$ & $1.610 \pm 0.341$ & $\boldsymbol{\nabla}$ \\
\hline $\begin{array}{c}\text { Western } \\
\text { medicine group }\end{array}$ & 10 & $0.242 \pm 0.126 \square \square$ & $1.527 \pm 0.362$ & $\mathbf{\square}$ \\
\hline
\end{tabular}

Note: The model group compares with the blank control group $\Delta \mathrm{P}<0.01$; Yanlingdan group and western medicine group compare with the model group $\mathrm{P}<0.01 ; \boldsymbol{\nabla} \mathbf{P}<0.05$; Yanlingdan group compares with the western medicine group $\nabla \mathrm{P}>0.05$.

\section{Conclusion}

Ovarian development is a process of continuous change, in which granular cells and oocytes begin to interact with each other. Granular cells supply $85 \%$ of required nutrients for oocyte development $[5,6]$. It has been proved[7-10] that granular cell apoptosis occurs during the process of follicular 
atresia and the granular cell apoptosis is an important and direct reason which leads to follicular atresia. The system belongs to the tumor necrosis factor receptor family and the FASL is the only natural ligand of it. At present, the signal path of cell apoptosis, which is mediated by Fas/FasL system, is one of the important ways of cell apoptosis. The results show that FAS and FASL proteins are weakly expressed in ovarian granular cells of the normal control group, but the model group was the opposite. The expression of the two of model group is enhanced. After the treatment, the expression intensity of gene proteins of Yanlingdan group and western medicine group is decreased, but the intensity is between the normal control group and the model group. There are significant differences between them. From it we can see that Yanlingdan can adjust the apoptosis gene proteins of ovarian granular cells. Its mechanism of action may be to disturb and restrain cell apoptosis which is induced by signal transfer system and prevent the open of dead passage through increasing the expression of apoptosis-suppressing gene FAS protein in ovarian granular cells so as to restrain the apoptosis of ovarian granular cells, promote the secretion of oestrogen and progestin and improve the endocrine function of ovary.

\section{Acknowledgements}

Science and technology planning project in Qiqihar city, Heilongjiang Province. (SFGG-201565): Influence of Yanling dan Prescription on the Apoptosis of Ovarian Granular Cells in Mice with Premature Ovarian Failure

\section{References}

[1].J.L.Cao, Q.Ding and so on: Experimental Research on Intervening EGF with Yougui Wan in Mice with Autoimmune Premature Ovarian Failure[J], Chinese Journal of Clinical Research, 2014, 6(31):3-6.(In Chinese)

[2].J.F.Yan, L.Hao, D.F.Zhang and so on: Effects of Tripterygium Wilfordii Polyglycosidium and Estrogen-progestogen Replacement Therapy on Expressions of Fas and fasl in Mouse Ovary[J]Journal of China Medical University, 2012, 8(41):688-691.(In Chinese)

[3].H.Y.Sun, Y.Z.Chen and X.O.Xue: The Effects of Siwu Mixture on Ovarian Granulose Cell Apoptosis of Chemotherapy-Induced Rats[J], Chinese Journal of Experimental Traditional Medical Formulae, 2012, 18 (10):256-259.(In Chinese)

[4].H.H.Cai, X.F.Fu, X.W.Ren and so on: Expression of Ki- 67 and estrogen receptor in the uterus of mice with autoimmune premature ovarian failure induced by peptide zona pellucida $3[\mathrm{~J}]$, Journal of Southern Medical University, 2015, 35(7): 992-997.(In Chinese)

[5].L.Zhu, S.P.Luo and L.M.Xu: Effects of Zuogui pill on Immune Premature Ovarian Failure in Mice Ovarian Ultrastruc-ture[J], Lishizhen Medicine and Materia Medica Research, 2016, 27(1): 42-44.(In Chinese)

[6].X.W.Ren, Y.L.He, H.H.Cai and so on: Expression of $\mathrm{Cx} 43$ and Bcl-2 in the Immunological Premature Ovarian Failure[J].Journal of Practical Obstetrics and Gynecology, 2016, 32(7) 515-517.(In Chinese)

[7].H.D.Wang, X.X.Zhu, S.H.Yan and so on: Effect of Bushen Jianpi on Ovarian Granulosa Cell Hormone Levels and Cell Cycle[J], Research and Practice on Chinese Medicines, 2016, 30(2): 21-23.(In Chinese)

[8].P.Liu, X.C.Li, Y.X.Yao and so on: The Regulating Factors of Apoptosis of Ovary Granular Cells[J], Progress in Modern Biomedicine, 2009, 9(13): 2562-2564.(In Chinese)

[9].[S.W.Yang, X.F.Sun, Y.Y.He and so on: Effect of Zuogui Pills on Ovarian Function in Mice with Chemotherapy-induced Premature Ovarian Failure[J],Chinese Traditional Patent Medicine, 2016, 38(4): 717-722.(In Chinese)

[10]. F.W.Wang, J.F.Wang and J.Xu: Cell Signaling Pathways Involved in the Regulation of Primordial Follicle Stimulating Processes[J], Reproduction and Contraception, 2016, 36(3): 208-239.(In Chinese) 